\title{
SUFFICIENCY IN A DISCONTINUOUS VARIATIONAL PROBLEM ALLOWING FOR CERTAIN BOUNDARY SUBARCS*
}

\author{
By G. T. MCALLISTER (Lehigh University)
}

1. Introduction. Let $\phi(x, y)$ be a sufficiently smooth function with $\phi_{y} \neq 0$ for all values of its argument. Let the regions $R_{\phi}^{+}$and $R_{\phi}^{-}$be defined to consist of all points $(x, y)$ for which $\phi(x, y) \geq 0$ and $\phi(x, y) \leq 0$, respectively.

Let $f^{+}(x, y, w)$ and $f^{-}(x, y, w)$ be given functions that are twice continuously differentiable functions of their arguments. Let $\left(x_{1}, y_{1}\right)$ and $\left(x_{2}, y_{2}\right)$ be given points in the plane. We consider the following variational problem: Find the minimum of the functional

$$
J(y)=\int_{x_{2}}^{x_{2}} f\left(x, y, y^{\prime}\right) d x,
$$

where $f\left(x, y, y^{\prime}\right)=f^{+}\left(x, y, y^{\prime}\right)$ for $(x, y) \varepsilon R_{\phi}^{+}$and $f\left(x, y, y^{\prime}\right)=f^{-}\left(x, y, y^{\prime}\right)$ for $(x, y) \varepsilon R_{\phi}^{-}$ among the class of functions in class $\left(C^{1}, D^{0}\right)$, with corners only on the interface, and which satisfy the end conditions $y\left(x_{1}\right)=y_{1}, y\left(x_{2}\right)=y_{2}$.

The problem of establishing necessary conditions and sufficient conditions was first treated by Bliss and Mason [1]. In that problem they assumed that the test curve intersected the boundary in exactly one point. This paper shall consider sufficiency when a test curve is allowed to have a subarc in common with the boundary $\phi(x, y)=0$.

An example of a physical phenomenon in which this case arises is that of the passage of light from one inhomogenous medium $R_{\phi}^{+}$into another medium $R_{\phi}^{-}$. For certain refraction indices, $\mu(x, y)$, the light ray may have a subarc on the interface $\phi(x, y)=0$.

It is to be observed that on the locus of discontinuity $\phi(x, y)=0$ there is an ambiguity as to the value of $f\left(x, y, y^{\prime}\right)$. However, if such a test curve is to render $J(y)$ a minimum, then it must do the same on any subinterval. In particular, along the boundary subarc the value of $f\left(x, y, y^{\prime}\right)$ must be taken to be such that

$$
f\left(x, y, y^{\prime}\right)=\min \left\{f^{+}\left(x, y, y^{\prime}\right), f^{-}\left(x, y, y^{\prime}\right)\right\},
$$

where $\left(x, y, y^{\prime}\right)$ is an element belonging to the locus of discontinuity.

For ease of presentation the following terminology shall be introduced: $A$ boundary subarc belongs to $f^{+}$means that for a subinterval of the abscissas of a boundary subarc we have that $\min \left\{f^{+}, f^{-}\right\}=f^{+}$with the arguments of the functions the coordinates in $\left(x, y, y^{\prime}\right)$-space of the subarc in question. In a similiar fashion we define a boundary subarc belongs to $f^{-}$.

2. Sufficient conditions. It is clear from the preceding discussion that those points $\left(x_{0}, y_{0}, y_{0}^{\prime}\right)$ belonging to the boundary subarc for which $f^{+}=f^{-}$are of special interest. We shall call such points cross-over points. At such points a boundary subarc may go from belonging to $f^{+}$to one belonging to $f^{-}$.

The following necessary conditions are easily derivable from Bliss and Mason [1]:

I. (1) For subarcs in $R_{\phi}^{+}$and $R_{\phi}^{-}$the Euler equation is satisfied using $f^{+}$and $f^{-}$, respectively.

${ }^{*}$ Received April 7, 1965; revised manuscript received May 20, 1965. Research done at Aberdeen Proving Ground, Maryland. 
(2) At corners, on the boundary, we have

$$
f^{+}-y_{+}^{\prime} f_{y^{\prime}}^{+}-f^{-}+y_{-}^{\prime} f_{y^{\prime}}^{-}=-y_{-}^{\prime}\left(f_{y^{\prime}}^{+}-f_{y^{\prime}}^{-}\right),
$$

where the arguments of $f^{+}$and $f^{-}$are $\left(x_{0}, y_{0}, y_{+}^{\prime}\right)$ and $\left(x_{0}, y_{0}, y_{-}^{\prime}\right)$, respectively, with $y_{-}^{\prime}$ and $y_{+}^{\prime}$ denoting the value of the slope before and after transition, respectively, with $\phi\left(x_{0}, y_{0}\right)=0$. Eq. (3) is for transition from an arc belonging to $f^{-}$to an arc belonging to $f^{+}$.

(3) Along a boundary subarc of a test curve we must have

$$
f=\min \left\{f^{+}, f^{-}\right\}
$$

with the arguments belonging to the boundary subarc.

(4) For subarcs belonging to $f^{+}$or $f^{-}$the Lagrange multipliers must satisfy $\lambda \leq 0$ or $\lambda \geq 0$, respectively. Moreover, entrance into the boundary (i.e., for an interval to the right of the abscissa of contact the boundary belongs to $f^{+}$) and exit from the boundary (i.e., for an interval to the left of the abscissa of the exit point the boundary belongs to $f^{-}$) require tangency.

IV. No point conjugate to $\left(x_{1}, y_{1}\right)$ lies between $x_{1}$ and the first point of contact with the interface. Similarly, there is no point conjugate to the last point of contact and $x_{2}$.

The necessary Condition II of Weierstrass and Condition III of Legendre are also derivable.

For sufficiency we modify Condition I (4) by requiring strict inequality in $\lambda$ and call this Condition I $\left(4^{\prime}\right)$. Condition IV is modified, and called Condition IV', to include the end-points. Condition III is modified to Condition III', i.e., $f_{y^{\prime} y^{\prime}}^{+}$and $f_{y^{\prime} y^{\prime}}^{-}$are never zero for points $(x, y, p)$, where $p$ is arbitrary and $\phi(x, y)=0$. It shall be assumed, for definiteness, that these derivatives are positive.

We will now catalogue the basic types of transition. Let $\xi_{1}$ and $\xi_{2}$ denote the abscissa of the first and last contact points with the boundary. Let $\eta$ denote a crossover point, $\xi_{1} \leq \eta \leq \xi_{2}$. We shall assume that $R_{\phi}^{-}$contains $\left(x_{1}, y_{1}\right)$ and $R_{\phi}^{+}$contains $\left(x_{2}, y_{2}\right)$. The following transitions are representative:

$(\alpha)$ The test curve enters the boundary at $\xi_{1}$, has a crossover point at $\eta>\xi_{1}$, and exists from the boundary at $\xi_{2}>\eta$.

$(\beta)$ The test curve intersects the boundary at $\xi_{1}$, belongs to $f^{+}$and exists at $\xi_{2}$.

More complicated transitions are possible but they will be variations of the cases that are treated above.

A theorem, basic to a proof of sufficiency, will now be stated and proved for case $(\alpha)$ :

Theorem. Let Conditions I(1), I(2), I $(3), \mathrm{I}\left(4^{\prime}\right)$, and II be satisfied. Let III" hold for all $(x, y)$ such that $\phi(x, y)=0$ and $x_{1} \leq x \leq x_{2}$. Then there exists a field in a neighborhood of a test curve that contains a boundary subarc.

Proof. In Fig. 1, the curves $C_{\xi_{1}}, C_{\xi_{2}}, C_{\eta}^{-}$, and $C_{\eta}^{+}$are subarcs of extremals of $f^{+}$ or $f^{-}$, depending on whether they lie in $R_{\phi}^{+}$or $R_{\phi}^{-}$, respectively, that are tangent to $\phi(x, y)=0$ with the indicated initial points and the indicated directions. The curves $C_{1}$ and $C_{4}$ are region extremals. The curves $C_{2}$ and $C_{3}$ are the boundary subarcs before and after crossover, respectively.

A neighborhood, in $R_{\phi}^{-}$, of the composite $C_{1}$ and $C_{2}$ (extending to $C_{\eta}^{-}$) may be simply covered by extremals of $f^{+}$. The region between $C_{\xi_{1}}$ and $C_{\eta}^{-}$is covered by extremals of 


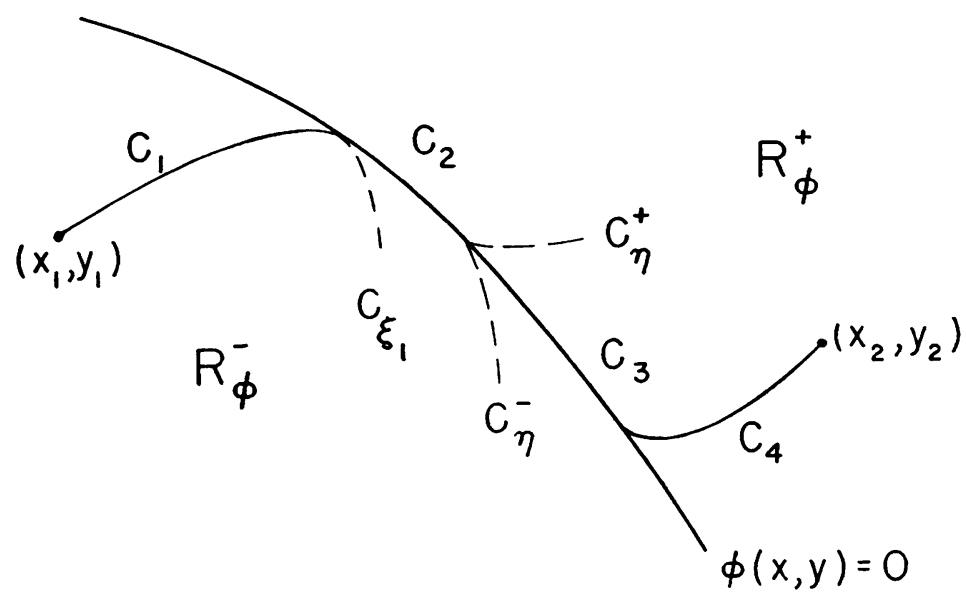

FIg. 1

$f^{+}$issuing from $\mathrm{C}_{2}$ on a tangent. That this covering exists and is simple was proved in Bliss [2].

A neighborhood, in $R_{\phi}^{+}$, of $C_{2}$ between a point near $(\xi, y(\xi))$ and $C_{\eta}^{+}$will be covered by extremals issuing from the boundary with slope $y_{+}^{\prime}$ given by a solution to Eq. (3) when we take $y_{-}^{\prime}$ as the slope of $\phi(x, y)$ at the point considered. That such solutions to Eq. (3) exist must be proved.

Let us take a point $\left(x_{0}, y_{0}\right)$ on the boundary such that the boundary slope $y_{-}^{\prime}=0$. This may be accomplished by a simple rotation. Then Eq. (3) becomes

$$
f^{+}(p)-p f_{p}^{+}(p)=f^{-}(0)
$$

where $p=y_{+}^{\prime}$. The derivative of the left-hand side of Eq. (4) is equal to $-p f_{p p}^{+}$. Condition III" and continuity shows that the left-hand side of Eq. (4) assumes all values. Hence, Eq. (4) has at least one solution. Eq. (4) represents a line tangent to the graph of $f^{+}(p)$ and passing through the point $\left(0, f^{-}(0)\right)$. By Condition III $^{\prime \prime}$, there will be at most two such tangents. Two tangents will exist when $\left(0, f^{+}(0)\right)$ is not on or above the graph of $f^{+}(p)$; only one tangent will occur when $\left(0, f^{-}(0)\right)$ is on the graph of $f^{+}(p)$. Of the two possible choices only one will bring an extremal into $R_{\phi}^{-}$.

Eq. (4) shows that $y_{+}^{\prime}$ will depend continuously on the coordinates of a boundary point $(x, y)$. Hence, $y_{+}^{\prime}$ approaches the slope of $C_{\eta}$ as the coordinates of the boundary approach the cross-over point.

We must now show that this covering is simple. We first observe that the coordinates of the boundary points and the slope of the boundary at those points may be made to depend, in a continuously differentiable way on a parameter $\mu$. Then the solution to the Euler equation for $f^{-}$may be written as $y_{+}^{\prime}(x, \mu)$ with $y_{+}^{\prime}(\mu, \mu)$ a solution of Eq. (4). Now $\partial y_{+}^{\prime} / \partial \mu$, at $x=\mu$, is non-zero, by $\mathrm{I}\left(4^{\prime}\right)$. Hence, continuity considerations give a simply covered neighborhood of $C_{2}$ in $R_{\phi}^{+}$.

In a similar fashion a neighborhood of $C_{3}$ in $R_{\phi}^{+}$is covered by extremals issuing from $C_{3}$ with the direction of the tangent to $C_{3}$. The strengthened Jacobi condition insures that this will simply cover $C_{4}$ and will extend to a neighborhood of the point of contact of $C_{4}$ and the interface. A neighborhood of $C_{3}$ in $R_{\phi}^{-}$is covered by extremals issuing from $C_{3}$ with slope satisfying the corner condition. 
Remarks. (1) In case $(\beta)$ there is no simple covering, unless $f^{+}$and $f^{-}$satisfy very special conditions. The reason for this is that any extremal close to the test curve will not have a solution of Eq. (4) given by the slope of the boundary at the point of intersection. Hence it must continue into the other region with a slope not tangent to the boundary. However, if we are to leave the boundary, at a point after initial contact, then we must leave on a tangent. Therefore, there is no unique way for an extremal to go to any point in the neighborhood of the boundary in the region $R_{\phi}^{+}$. Such situations must simply be compared with extremals that satisfy the boundary conditions and contain no boundary subarc.

(2) The entire discussion of boundary subarcs is eliminated if one assumes that $f^{+}(x, y, p) \neq f^{-}(x, y, p)$, where $\xi_{1} \leq x \leq \xi_{2}, \phi(x, y)=0$, and $p$ is arbitrary. This is certainly the situation when light passes from one homogenous medium into another.

In order to complete a discussion of sufficiency, it must be shown that the composite curve $C_{1}, C_{2}, C_{3}$, and $C_{4}$, to be denoted by $C$, renders $J(y)$ a minimum among all curves in the neighborhood which was constructed in the last theorem. This will be accomplished by extending the method of Weierstrass as developed in Bliss [2].

THEOREM 2. If the assumptions of the last theorem are satisfied and if the Weierstrass $E$-functions are positive in their respective sides of the boundary then any curve $V$ satisfying the boundary conditions and lying in the neighborhood of $C$ satisfies the relation

$$
J(V)>J(C) .
$$

Proof. Let $V$ be a curve in the $R_{\phi}^{-}$portion of the neighborhood constructed above. Let the graph of $V$ be given by $y=g(x)$. Assume that $V$ intersects the boundary at a point $\xi_{2}^{\prime}>\eta$; the case $\xi_{2}^{\prime} \leq \eta$ has been treated in Bliss [2]. Let the boundary be given by $y=\beta(x)$.

We define a function $W\left(x^{\prime}\right)$ given by

$$
W^{\top}\left(x^{\prime}\right)=J_{\nu}(C)+J_{p}\left(V^{\prime}\right),
$$

where $p$ is the slope function of a point with abscissa $x^{\prime}$, lying on $V$, and $J_{p}(C)$ is an integral along $C$ leaving the boundary at $\xi, \beta(\xi)$, on the extremal that intersects $V$ at $\left(x^{\prime}, g\left(x^{\prime}\right)\right) ; J_{p}(V)$ has a similar meaning. Differentiation of (5) with respect to $x^{\prime}$ leads to

$$
\begin{aligned}
\frac{d W}{d x^{\prime}}=\left[f^{-}\left(x, \beta, \beta^{\prime}\right)\right. & \left.-f^{-}\left(x, y_{+}, y_{+}^{\prime}\right)\right]_{x=\xi} \frac{d \mu}{d x^{\prime}}+\left.\frac{d \mu}{d x^{\prime}} \frac{\partial y_{+}^{\prime}}{\partial \mu} f_{y^{\prime}}^{-}\left(x, y_{+}, y_{+}^{\prime}\right)\right|_{x=x^{\prime}} \\
& -\left.\frac{d \mu}{d x^{\prime}} \frac{\partial y_{+}^{\prime}}{\partial \mu} f_{y^{\prime}}^{-}\left(x, y_{+}, y_{+}^{\prime}\right)\right|_{x=\xi}+\left[f\left(x, y_{+}, y_{+}^{\prime}\right)-f\left(x, g, g^{\prime}\right)\right]_{x^{\prime} x^{\prime}},
\end{aligned}
$$

where $\mu$ is the parameter introduced in the last theorem. Observing that $d \mu / d x^{\prime}$, at $x=\mu$, is 1 , we obtain

$$
\frac{d W}{d x^{\prime}}=-\left.E\left(x, y, y_{+}, y_{+}^{\prime}, g, g^{\prime}\right)\right|_{x=x^{\prime}}-\left.E\left(x, y, y_{+}, y_{+}^{\prime}, \beta, \beta^{\prime}\right)\right|_{x=\xi},
$$

where $E$ is the Weierstrass $E$-function; the expression ( 7$)$ is obtained by combining the second and fourth terms of Eq. (6), and the first and third terms of Eq. (6). Our hypothesis shows that $d W / d x^{\prime}$ is decreasing.

The exceptional cases are treated as in Bliss [2].

The same treatment extends to a discussion of the region about $C$ in $R_{\phi}^{+}$. 


\section{REFERENCES}

1. G. A. Bliss and M. Mason, A problem of the calculus of variations in which the integrand is discontinuous, Trans. Amer. Math. Soc. 7 (1906), p. 325

2. G. A. Bliss, Sufficient conditions for a minimum with respect to one-sided variations, Trans. Amer. Math. Soc. 5 (1904), p. 477 